\title{
Keynote
}

\section{Extreme Visual Languages}

\author{
Shi-Kuo Chang \\ Professor \\ School of Computer and Information \\ University of Pittsburgh \\ USA
}

\begin{abstract}
Extreme Visual Languages are visual languages that can communicate an integrated/holistic meaning of an application, a philosophy or even the entire spatial/temporal dimensions of a universe in a single or a few visual expressions. An example is the aliens' visual language in the movie "The Arrival" where each visual sentence expresses the continuum of space and time. Another example is a visual diagram illustrating the spread of Covid-19 in the world. A third example is the Ying/Yang icon that appears in the national flag of the Republic of Korea (and many other places). A fourth example is the red flag. A natural extension to include sound and other media leads to Extreme Multimedia Languages. In this talk I will discuss extreme visual and multimedia languages, both abbreviated as $X V L s$, including empirical and comparative studies of existing XVLs and the investigation of syntax, semantics, dynamics and application integration of existing and/or new XVLs and related topics.
\end{abstract}

\section{About the Speaker}

Dr. Chang is the founder and President of KSI Research. Dr. Chang received the B.S.E.E. degree from National Taiwan University in 1965. He received the M.S. and Ph.D. degrees from the University of California, Berkeley, in 1967 and 1969, respectively. He was a research scientist at IBM Watson Research Center from 1969 to 1975. From 1986 to 1990, he was Professor and Chairman of the Department of Computer Science, University of Pittsburgh. He is currently Professor and Director of Center for Parallel and Distributed Systems, University of Pittsburgh. Dr. Chang is a Fellow of IEEE. He has been consultant for IBM, Laboratories, Standard Oil, Honeywell, and Naval Research Laboratory. His research interests include knowledge-based systems, pictorial information systems, visual languages and computer vision. Dr. Chang has published over two hundred papers, and written or edited eight books. His books, Principles of Pictorial Information Systems Design, (Prentice-Hall, 1989), and Principles of Visual Programming Systems, (Prentice-Hall, 1990), are pioneering advanced textbooks in these research areas.

Dr. Chang is the Editor of the Journal of Visual Language and Computing published by KSI Research, and the Editor of the International Journal of Software Engineering \& Knowledge Engineering published by World Scientific Press. 